\title{
TOPOLOGICAL MIXING OF HIGHER DEGREES
}

\author{
SUE GOODMAN ${ }^{1}$ AND BRIAN MARCUS ${ }^{2}$
}

\begin{abstract}
We give examples of homeomorphisms which are topologically 1-mixing but not topologically 2-mixing. One is a subshift and the other is a diffeomorphism of the torus.
\end{abstract}

Introduction. The question of whether measure-theoretic mixing implies mixing of all degrees has been an open problem in ergodic theory for many years [1]. This note deals with the analogous notions (which are much cruder) in topological dynamics.

Let $T: X \rightarrow X$ be a homeomorphism of a compact metric space. One says that $T$ is 1-mixing if: for all nonempty open sets $U, V$, there exists $M$ such that if $m \geqslant M$ then $T^{m}(U) \cap V \neq \varnothing$. One says that $T$ is 2-mixing if: for all nonempty open sets $U, V$, and $W$, there exists $M$ such that if $m_{1}, m_{2} \geqslant M$ then $T^{m_{2}}\left(T^{m_{1}}(U) \cap V\right) \cap W \neq \varnothing$. For continuous one-parameter flows, one has the obvious analogous definitions.

It is clear that 2-mixing implies 1-mixing. In this note, we give two very simple examples which both show that 1-mixing does not imply 2-mixing. This has also been done by M. Dekking and M. Keane [3]. Their example is a substitution minimal set, while both of our examples have a fixed point. None of these examples is measure-theoretically mixing.

Our first example is a subshift (i.e., a symbolic dynamical system). It has exactly two ergodic invariant measures. One is the point mass at the fixed point and the other is a (measure-theoretically) weakly mixing, but not strongly mixing, measure which is positive on open sets.

Our second example is a continuous 1-parameter flow (a Stepanoff flow [2]) on the 2-dimensional torus. The orbits of this flow are the same as the orbits of an irrational flow except that exactly one orbit of the latter splits into three orbits of the former: a fixed point and two half-lines which die at the fixed point, one in forward time and the other in backward time. Since the flow will be 1-mixing but not 2-mixing, it is easy to see that the same holds for every nonzero time $t$ map. Thus, we get a homeomorphism of the torus which is 1-mixing but not 2-mixing. With a more careful analysis, one can make the flow smooth and, hence, get a diffeomorphism of the torus which is 1-mixing but not 2-mixing.

Received by the editors January 31, 1977 and, in revised form, September 11, 1977.

AMS (MOS) subject classifications (1970). Primary 54H20, 34C35.

Key words and phrases. Topological mixing, higher degrees, subshift, Stepanoff flow.

'Partially supported by NSF grants MCS76-05988 and MCS72-05055 A04.

${ }^{2}$ Partially supported by NSF grant MCS76-05969. 
We mention that while both our examples have zero entropy, one can make positive entropy examples by simply taking the direct product of one of our maps with any positive entropy, 1-mixing map (e.g., the full shift on two symbols).

Finally, the example in $\$ 1$ can be generalized to give examples of $n$-mixing maps which are not $(n+1)$-mixing (with the obvious definitions). Since this paper was written, B. Weiss has found even simpler symbolic examples.

We thank R. Adler for helpful discussions.

1. The symbolic example. Let $\{0,1\}$ denote the two-point space with the discrete topology. Let $\Sigma=\{0,1\}^{Z}$. A point $\mathbf{x} \in \Sigma$ is a bi-infinite sequence of 0 's and 1's, $\mathbf{x}=\cdots x_{-2} x_{-1} x_{0} x_{1} x_{2} \cdots$. The shift homeomorphism $T: \Sigma \rightarrow$ $\Sigma$ is defined by

$$
T(\mathbf{x})=\mathbf{y} \quad \text { where } y_{i}=x_{i+1} .
$$

If $\Lambda \subset \Sigma$ is a closed $T$-invariant subset, then $\left.T\right|_{\Lambda}$ is called a subshift. One way to construct a subshift is to take the $T$-orbit-closure of a point $\mathbf{x} \in \Sigma$. The following characterizes mixing properties of these subshifts in terms of the blocks $\left(c_{1} \cdots c_{n} \in\{0,1\}^{n}\right)$ which appear in $\mathbf{x}$.

Proposition 1. Let $\Lambda$ be the T-orbit-closure of $\mathbf{x}$. Then

(a) $\left.T\right|_{\Lambda}$ is 1-mixing if and only if: for all finite blocks $c_{1} \cdots c_{n}$ which appear in $\mathbf{x}$, there exists $M$ such that if $m \geqslant M$ then one can find $i \in Z$ such that

$$
x_{i}=c_{1}, \quad x_{i+1}=c_{2}, \ldots, \quad x_{i+n-1}=c_{n}
$$

and

$$
x_{i+m}=c_{1}, \quad x_{i+m+1}=c_{2}, \ldots, \quad x_{i+m+n-1}=c_{n} .
$$

(b) $\left.T\right|_{\Lambda}$ is 2-mixing if and only if: for all finite blocks $c_{1} \cdots c_{n}$ which appear in $\mathbf{x}$, there exists $M$ such that if $m_{1}, m_{2} \geqslant M$ then one can find $i \in Z$ such that

$$
\begin{aligned}
x_{i} & =c_{1}, \quad x_{i+1}=c_{2}, \ldots, \quad x_{i+n-1}=c_{n}, \\
x_{i+m_{1}} & =c_{1}, \quad x_{i+m_{1}+1}=c_{2}, \ldots, \quad x_{i+m_{1}+n-1}=c_{n}
\end{aligned}
$$

and

$$
x_{i+m_{1}+m_{2}}=c_{1}, \quad x_{i+m_{1}+m_{2}+1}=c_{2}, \ldots, \quad x_{i+m_{1}+m_{2}+n-1}=c_{n} .
$$

The proof of this proposition is straightforward and well known; it follows from the fact that each block represents an open set and the collection of these open sets forms a basis for the topology.

Let $a_{0}=0$ and, inductively, $a_{n}=a_{n-1} b_{n} a_{n-1}$ (juxtaposition), where $b_{n}$ is the block consisting of $n$ consecutive l's. Observe that if $k>n$, then $a_{n}$ is an initial block of $a_{k}$. Thus, it makes sense to define $\mathbf{x}^{+}$as the one-sided infinite sequence of 0's and 1's which has each $a_{n}$ as an initial block. Define $\mathbf{x}$ by

$$
x_{i}= \begin{cases}x_{i}^{+} & \text {if } i \geqslant 0, \\ 1 & \text { if } i<0 .\end{cases}
$$

Proposition 2. Let $\Lambda$ be the $T$-orbit-closure of $\mathbf{x}$. Then $\left.T\right|_{\Lambda}$ is 1-mixing but not 2-mixing. 
Proof. First observe that for every $n$ we can write the point $\mathbf{x}^{+}$as follows:

$$
\mathbf{x}^{+}=a_{n-1} b_{i_{1}} a_{n-1} b_{i_{2}} a_{n-1} b_{i_{3}} \cdots
$$

where

$$
\begin{array}{ll}
i_{j}=n & \text { if } j \text { is odd } \\
i_{j}>n & \text { if } j \text { is even. }
\end{array}
$$

Moreover, $\left\{i_{j}\right\}_{j} \supset[n,+\infty)$.

This implies that Proposition 1(a) holds for every block which is contained in some $a_{n-1}$. But this includes every block which occurs in $\mathbf{x}$. So, $\left.T\right|_{\Lambda}$ is 1-mixing.

We show that $\left.T\right|_{\Lambda}$ is not 2-mixing for the $a_{0}$-block. To do this, it suffices to show that there is an infinite sequence $k_{n} \rightarrow \infty$ such that in $\mathbf{x}$ one never sees a block of the form

$$
x_{i} x_{i+1} \cdots x_{i+k_{n}} \cdots x_{i+2 k_{n}},
$$

where $x_{i}=x_{i+k_{n}}=x_{i+2 k_{n}}=0$. We claim that if $k_{n}=\operatorname{length}\left(a_{n-1}\right)+$ length $\left(b_{n}\right)-1$, then $\left\{k_{n}\right\}$ is such a sequence.

Suppose that a block of the form $(* *)$ appears in $\mathbf{x}$. We seek a contradiction. Since there are no zeros in the $b$-blocks, $x_{i}$ must occur in one of the $a_{n-1}$ blocks in (*) above. But then by the choice of $k_{n}$, it is easy to see that $x_{i+k_{n}}$ must appear in the next $a_{n-1}$ block to the right. And $x_{i+2 k_{n}}$ must appear in the next $a_{n-1}$ block after that. But then one of the intervening blocks $b_{i}$ must be $b_{n}$. For definiteness, let us say that it is the $b$ block in between $x_{i}$ and $x_{i+k_{n}}$. (The other case may be treated in exactly the same manner.) But then it is not hard to see, from the definition of $k_{n}$, that $x_{i+k_{n}}=x_{i-1}$. Thus, both $x_{i-1}$ and $x_{i}$ are zero. But from the construction of the sequence $\mathbf{x}$, it is evident that there are never two zeros in a row.

2. The Stepanoff flow. We shall now give an example of a continuous flow on the torus $T^{2}$ which is 1 -mixing, but not 2-mixing. The example is described in the introduction and is called a Stepanoff flow. Oxtoby observed [2] that since the two half-lines (involved in the definition) are dense, every continuous parametrization of this flow is 1 -mixing. ${ }^{3}$ We shall produce a continuous parametrization which is not 2-mixing. We start with just the orbits of a Stepanoff flow, and will inductively define a parametrization.

Fix a flow box $F=I \times I$ (for the original irrational flow) which contains the fixed point $(0,0)$ of the Stepanoff flow. (Note: $I=[-1,1]$.) We assume that the flow moves from left to right. Fix a closed disk $A \subset F^{c}$. Let $\varepsilon_{n} \downarrow 0$ and $F_{n}=I \times\left(-\varepsilon_{n}, \varepsilon_{n}\right)$. We assume that outside $F_{1}$, the flow is defined by the unit speed parametrization. Now we describe how to define the parametrization on $F_{n}-F_{n+1}$ assuming that it has been defined outside $F_{n}$. The condition will essentially involve only the "crossing time" function (i.e., the function $f: I \rightarrow(0,+\infty]$ which tells how long it takes to cross $I \times I)$.

\footnotetext{
${ }^{3}$ Simply observe that every open set meets the half-line which approaches the fixed point and thus gets stretched out along the half-line which emanates from the fixed point.
} 
For each $x \in T^{2}-\operatorname{int}\left(F_{n}\right)$, there is a smallest time $s(x) \geqslant 0$ such that $\varphi_{s(x)}(x) \in\{-1\} \times\left(-\varepsilon_{n}, \varepsilon_{n}\right)$. This makes sense since the parametrization is defined outside $F_{n}$. Let $S_{n}=\max _{x \in A}\{s(x)\}$. Note $S_{n}<\infty$ since $s(x)$ is a semicontinuous function on a compact set.

Choose $\delta_{n} \in\left(0, \varepsilon_{n}\right)$ so that if $\gamma \in\left(-\varepsilon_{n},-\delta_{n}\right) \cup\left(\delta_{n}, \varepsilon_{n}\right)$ and $x=(1, \gamma)$, then

$$
\varphi_{s(x)}(x) \in\{-1\} \times\left\{-\delta_{n}, \delta_{n}\right\} .
$$

Assume also that $\delta_{n}>\varepsilon_{n+1}$.

Extend the parametrization to $F_{n}-F_{n+1}$ so that the crossing time function is monotonic on each side of zero and is at least $S_{n}$ on $\left(I \times\left[-\delta_{n}, \delta_{n}\right]\right) \cap$ $F_{n+1}^{c}$.

The induction process gives a continuous parametrization on $T^{2}-(I \times$ $\{0\}$ ). It is easy to see that with a little care ${ }^{4}$ (in the induction process) this parametrization extends continuously to all of $T^{2}$ in such a way that the resulting flow is a Stepanoff flow. The important point is that with such a parametrization the crossing time across each $I \times\left[-\delta_{n}, \delta_{n}\right]$ is at least $S_{n}$. Note also that there are many such parametrizations.

In fact, Jenny Harrison has shown that flows with this type of singularity are smoothable-in the sense that there is a homeomorphism $h$ such that the flow $h \circ \phi_{s} \circ h^{-1}$ is smooth.

Proposition 3. Given any Stepanoff flow $\left\{\varphi_{s}\right\}$ parametrized as described, $\left\{\varphi_{s}\right\}$ is not 2-mixing.

Proof. Let $A, F_{n}$ be as above. We claim that $\varphi_{S_{n}}\left(\varphi_{S_{n}}(A) \cap A\right) \subset F_{n}$, hence $\varphi_{S_{n}}\left(\varphi_{S_{n}}(A) \cap A\right) \cap A=\varnothing$. Observe $S_{n} \rightarrow \infty$. Clearly, this would prove the theorem.

Suppose $y \in A$ and $\varphi_{S_{n}}(y) \in A$. By time $S_{n}$, the positive semiorbit $\mathcal{O}$ from $y$ has entered $F_{n}$. But since it has returned to $A$ by this time, it must have entered $F_{n}$ along $\left[\{-1\} \times\left(\delta_{n}, \varepsilon_{n}\right)\right] \cup\left[\{-1\} \times\left(-\varepsilon_{n},-\delta_{n}\right)\right]$ where the crossing time can be less than $S_{n}$.

Let $\varphi_{t_{1}}(y)$ denote the first exit point (from $\left.F_{n}\right)$ for $\theta$, and let $\varphi_{t_{2}}(y)$ denote the next entrance point (into $F_{n}$ ). (See Figure 1.)

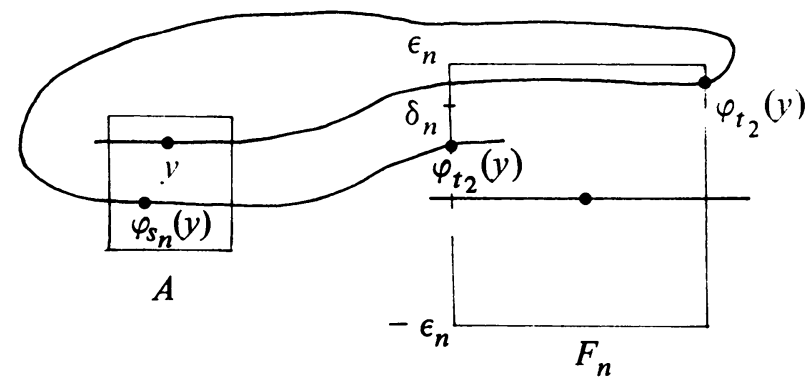

FIGURE 1

${ }^{4}$ Clearly the crossing times must approach $\infty$ as $n$ does. 
By the choice of $\delta_{n}, \varphi_{t_{2}}(y) \in\{-1\} \times\left(-\delta_{n}, \delta_{n}\right)$. Since $\varphi_{S_{n}}(y) \in A$ and hence is not in $F_{n}$, one sees that (as indicated in Figure 1) $t_{1}<S_{n}<t_{2}$. Thus, by the definition of $S_{n}$ (applied to $x=\varphi_{S_{n}}(y)$ ) and the fact that the minimum crossing time in $\{-1\} \times\left(-\delta_{n}, \delta_{n}\right)$ is at least $S_{n}$, we have $\varphi_{2 S_{n}}(y) \in F_{n}$ as desired.

\section{REFERENCES}

1. N. Friedman, Introduction to ergodic theory, Van Nostrand, New York, 1970, pp. 65-66.

2. J. C. Oxtoby, Stepanoff flows on the torus, Proc. Amer. Math. Soc. 4 (1953), 982-987.

3. F. M. Dekking and M. Keane, Mixing properties of substitutions (preprint).

School of Mathematics, Institute for Advanced Study, Princeton, New Jersey 08540

ibM Thomas J. Watson Research Center, Yorktown Heights, New York 10541

Department of mathematics, University of North Carolina, Chapel Hill, North CARolina 27514 (Current address of both authors) 